\title{
Mepolizumab Treatment in Patients with Severe Eosinophilic Asthma
}

\author{
Hector G. Ortega, M.D., Sc.D., Mark C. Liu, M.D., Ian D. Pavord, D.M., \\ Guy G. Brusselle, M.D., J. Mark FitzGerald, M.D., Alfredo Chetta, M.D., \\ Marc Humbert, M.D., Ph.D., Lynn E. Katz, Pharm.D., Oliver N. Keene, M.Sc., \\ Steven W. Yancey, M.Sc., and Pascal Chanez M.D., Ph.D., \\ for the MENSA Investigators*
}

ABSTRACT

From the Respiratory Therapeutic Area Unit, GlaxoSmithKline, Research Triangle Park, NC (H.G.O., L.E.K., S.W.Y.); Johns Hopkins Asthma and Allergy Center, Baltimore (M.C.L.); Respiratory Medicine Unit, Nuffield Department of Medicine, University of Oxford, Oxford (I.D.P.), and Clinical Statistics, GlaxoSmithKline, Stockley Park, Middlesex (O.N.K.) - both in the United Kingdom; the Department of Respiratory Medicine, Ghent University Hospital, Ghent, Belgium (G.G.B.); the Lung Centre, Institute for Heart and Lung Health, Vancouver, BC, Canada (J.M.F.); the Department of Clinical and Experimental Medicine, University of Parma, Parma, Italy (A.C.); and Assistance PubliqueHôpitaux de Paris, Département Hospitalo-Universitaire Thorax Innovation, Service de Pneumologie, Hôpital Bicêtre, Université Paris-Sud, and INSERM Unité Mixte de Recherche 999, Le KremlinBicêtre (M.H.), and Unités Mixtes de Recherche INSERM Unité 1067 Centre Nationale de la Recherche Scientifique 7733, Aix-Marseille Université, Department of Respiratory Diseases and Clinical Investigation Center, Assistance Publique-Hôpitaux de Marseille, Hôpital Nord, Marseille (P.C.) - both in France. Address reprint requests to $D r$. Ortega at GlaxoSmithKline, 5 Moore Dr., P.O. Box 5.3317.3A, Research Triangle Park, NC 27709, or at hector.g.ortega@gsk.com.

*A complete list of investigators in the Mepolizumab as Adjunctive Therapy in Patients with Severe Asthma (MENSA) study is provided in the Supplementary Appendix, available at NEJM.org.

This article was published on September 8 , 2014, at NEJM.org.

N EnglJ Med 2014;371:1198-207. DOI: 10.1056/NEJMoal403290

Copyright (C) 2014 Massachusetts Medical Society.

\section{BACKGROUND}

Some patients with severe asthma have frequent exacerbations associated with persistent eosinophilic inflammation despite continuous treatment with high-dose inhaled glucocorticoids with or without oral glucocorticoids.

\section{METHODS}

In this randomized, double-blind, double-dummy study, we assigned 576 patients with recurrent asthma exacerbations and evidence of eosinophilic inflammation despite high doses of inhaled glucocorticoids to one of three study groups. Patients were assigned to receive mepolizumab, a humanized monoclonal antibody against interleukin-5, which was administered as either a 75-mg intravenous dose or a 100-mg subcutaneous dose, or placebo every 4 weeks for 32 weeks. The primary outcome was the rate of exacerbations. Other outcomes included the forced expiratory volume in 1 second $\left(\mathrm{FEV}_{1}\right)$ and scores on the St. George's Respiratory Questionnaire (SGRQ) and the 5-item Asthma Control Questionnaire (ACQ-5). Safety was also assessed.

RESULTS

The rate of exacerbations was reduced by $47 \%$ (95\% confidence interval [CI], 29 to 61) among patients receiving intravenous mepolizumab and by 53\% (95\% CI, 37 to 65) among those receiving subcutaneous mepolizumab, as compared with those receiving placebo ( $\mathrm{P}<0.001$ for both comparisons). Exacerbations necessitating an emergency department visit or hospitalization were reduced by $32 \%$ in the group receiving intravenous mepolizumab and by $61 \%$ in the group receiving subcutaneous mepolizumab. At week 32, the mean increase from baseline in $\mathrm{FEV}_{1}$ was $100 \mathrm{ml}$ greater in patients receiving intravenous mepolizumab than in those receiving placebo $(\mathrm{P}=0.02)$ and $98 \mathrm{ml}$ greater in patients receiving subcutaneous mepolizumab than in those receiving placebo $(\mathrm{P}=0.03)$. The improvement from baseline in the SGRQ score was 6.4 points and 7.0 points greater in the intravenous and subcutaneous mepolizumab groups, respectively, than in the placebo group (minimal clinically important change, 4 points), and the improvement in the ACQ- 5 score was 0.42 points and 0.44 points greater in the two mepolizumab groups, respectively, than in the placebo group (minimal clinically important change, 0.5 points) $(\mathrm{P}<0.001$ for all comparisons). The safety profile of mepolizumab was similar to that of placebo.

\section{CONCLUSIONS}

Mepolizumab administered either intravenously or subcutaneously significantly reduced asthma exacerbations and was associated with improvements in markers of asthma control. (Funded by GlaxoSmithKline; MENSA ClinicalTrials.gov number, NCT01691521.) 
EVERE ASTHMA AFFECTS LESS THAN 10\% of patients with asthma and is associated with substantial morbidity and mortality and a large fraction of the health care costs among patients with asthma. ${ }^{1-3}$ Despite available care, recurrent asthma exacerbations are a major issue in a subgroup of patients with eosinophilic airway inflammation. ${ }^{4-6}$ Mepolizumab, a humanized monoclonal antibody against interleukin-5, selectively inhibits eosinophilic inflammation ${ }^{7,8}$ and reduces the number of eosinophils in both sputum and blood, resulting in a reduction in exacerbations and in the need for treatment with systemic glucocorticoids. ${ }^{7-12}$ In the Dose Ranging Efficacy and Safety with Mepolizumab (DREAM) study $^{13}$ of intravenous mepolizumab, investigators defined key phenotypic characteristics of the target population that were associated with a response to treatment with mepolizumab. In our study, called Mepolizumab as Adjunctive Therapy in Patients with Severe Asthma (MENSA), we used these key characteristics (i.e., blood eosinophil count, number of previous exacerbations, and dose of inhaled glucocorticoids) to identify eligible patients in a placebo-controlled comparison of subcutaneous and intravenous administration of mepolizumab. We sought to determine whether the use of anti-interleukin-5 therapy would mitigate the requirement for frequent glucocorticoid use in patients with severe asthma, most of whom did not yet require glucocorticoids on a daily basis. In another article in the Journal, Bel and colleagues ${ }^{14}$ report the results of a study in which they investigated whether the neutralization of interleukin-5 allows for reductions in the dose of oral glucocorticoids in patients who require long-term daily use.

METHODS

STUDY DESIGN AND OVERSIGHT

The MENSA study was a multicenter, randomized, double-blind, double-dummy, phase 3, placebocontrolled trial that was conducted from October 2012 through January 2014. The study consisted of a run-in period of 1 to 6 weeks, which was followed by a 32-week treatment phase and a followup 8-week safety phase (Fig. 1A).

The protocol, which is available with the full text of this article at NEJM.org, was developed by the sponsor, GlaxoSmithKline. The first draft of the manuscript was written by the first author, an employee of GlaxoSmithKline; editorial support funded by GlaxoSmithKline was provided by Gardiner-Caldwell Communications. Data were collected by the investigators and analyzed by employees of the sponsor. All the authors vouch for the accuracy and completeness of the data, the statistical analysis, and the fidelity of the study to the protocol. The protocol was approved by local or national research ethics committees.

\section{PATIENTS}

The study patients were between 12 and 82 years of age. All enrolled patients were required to have received a clinical diagnosis of asthma by a physician and to have a forced expiratory volume in 1 second $\left(\mathrm{FEV}_{1}\right)$ of less than $80 \%$ of the predicted value (in the case of adults) or an $\mathrm{FEV}_{1}$ of less than $90 \%$ of the predicted value or a ratio of the $\mathrm{FEV}_{1}$ to the forced vital capacity (FVC) of less than 0.8 (in the case of adolescents under the age of 18 years). In addition, patients were required to have one or more of the following three test results: $\mathrm{FEV}_{1}$ reversibility of more than $12 \%$, positive results on methacholine or mannitol challenge at visit 1 or 2 or during the previous year, and $\mathrm{FEV}_{1}$ variability ( $\geq 20 \%$ ) between two clinic visits in the past 12 months. All patients had to have had at least two asthma exacerbations in the previous year that were treated with systemic glucocorticoids while they were receiving treatment with at least $880 \mu \mathrm{g}$ of fluticasone propionate or the equivalent by inhalation per day and at least 3 months of treatment with an additional controller. In addition, all patients had to have an eosinophil count of at least 150 cells per microliter in the peripheral blood at screening or at least 300 cells per microliter at some time during the previous year. Patients were allowed to continue their current antiasthma therapy throughout the study. All patients provided written informed consent.

\section{STUDY INTERVENTIONS}

Patients were randomly assigned to receive mepolizumab, which was administered as either a 75-mg intravenous dose or a 100-mg subcutaneous dose, or placebo every 4 weeks for 32 weeks (Fig. 1A). Randomization was performed with the use of a centralized computer-generated, permuted-block schedule. The study drugs were prepared by staff members who were aware of the studygroup assignments but were not involved in study assessments. Mepolizumab and placebo were identical in appearance and were administered by a staff member who was unaware of the study- 
group assignments. Details regarding study measurements and procedures are provided in the Supplementary Appendix, available at NEJM.org.

\section{PRIMARY OUTCOME}

The primary outcome was the annualized frequency of clinically significant exacerbations, which were defined as worsening of asthma such that the treating physician elected to administer systemic glucocorticoids for at least 3 days or the patient visited an emergency department or was hospitalized. ${ }^{15}$ Exacerbations were confirmed by objective changes that patients recorded daily in an electronic diary (eDiary, PHT).

\section{STUDY MEASUREMENTS}

At each clinic visit, we evaluated results of spirometric and hematologic tests and administered the 5-item Asthma Control Questionnaire (ACQ-5), on which scores range from 0 to 6 , with higher scores indicating worse function, and 0.5 is the minimal clinically important difference between scores. At randomization and the final study visit, we administered the St. George's Respiratory Questionnaire (SGRQ), on which scores range from 0 to 100, with higher scores indicating worse function and a change of 4 units considered to be clinically relevant. Additional questionnaires included a survey in which the clinician asked patients to rate their global response to therapy (on a scale of 1 to 7 , with 1 indicating substantial improvement and 7 indicating substantial worsening of asthma).

Safety was evaluated by an assessment of adverse events, vital signs, electrocardiographic findings, and immunogenicity, along with clinical laboratory testing. Levels of antibodies against mepolizumab were measured before randomization and at three time points $(16,32$, and 40 weeks) after randomization.

\section{STATISTICAL ANALYSIS}

All patients who received at least one dose of a study drug were included in a modified intention-to-treat analysis. For the primary outcome, the rate of exacerbations was compared in the three groups with the use of a negative binomial model $^{16}$ that included covariates for treatment, use of maintenance oral glucocorticoids, geographic region, number of exacerbations in the previous year, and baseline percentage of the predicted $\mathrm{FEV}_{1}$. We estimated that with 180 patients in each group, the study would have a power of $90 \%$
Figure 1 (facing page). Study Design and Enrollment and Outcomes.

Panel A shows the design of the study. Patients who received $75 \mathrm{mg}$ of mepolizumab intravenously also received placebo subcutaneously, patients who received $100 \mathrm{mg}$ of mepolizumab subcutaneously also received placebo intravenously, and patients who received placebo received placebo both intravenously and subcutaneously. Panel B shows the screening, randomization, treatment, and follow-up of the patients.

to detect a $40 \%$ decrease in the exacerbation rate, from 2.40 per year in the placebo group to 1.44 per year in each of the mepolizumab groups, at a two-sided significance level of 0.05 . In performing this calculation, we assumed that the number of exacerbations would follow a negative binomial distribution ${ }^{17}$ with a dispersion parameter $\mathrm{k}=0.8$.

The two primary comparisons were of intravenous mepolizumab with placebo and of subcutaneous mepolizumab with placebo. For strong control of a type I error in multiple testing, we used a Hochberg procedure for treatment comparisons and a hierarchical testing procedure for primary and secondary outcomes. The reported $\mathrm{P}$ values are two-sided and have not been adjusted for multiple comparisons.

We used a mixed-model, repeated-measures method to analyze data regarding the $\mathrm{FEV}_{1}$, responses on questionnaires, and blood eosinophil counts; included in this model were the abovementioned covariates along with baseline value, visit, and terms for the interaction of visit with baseline value and of visit with treatment group. A prespecified log transformation was applied to blood eosinophil counts before analysis. We performed a post hoc analysis of the overall response to therapy, as rated by both patients and clinicians. We used a proportional-odds model (ordered multinomial logistic regression) to analyze the number of patients who evaluated themselves or were evaluated by a clinician in each category of response to therapy, with the above-mentioned covariates. In this analysis, patients with missing responses were included in the "significantly worse" category.

\section{RESULTS}

\section{PATIENTS}

Of the 802 patients who were screened, 576 underwent randomization and constituted the modi- 


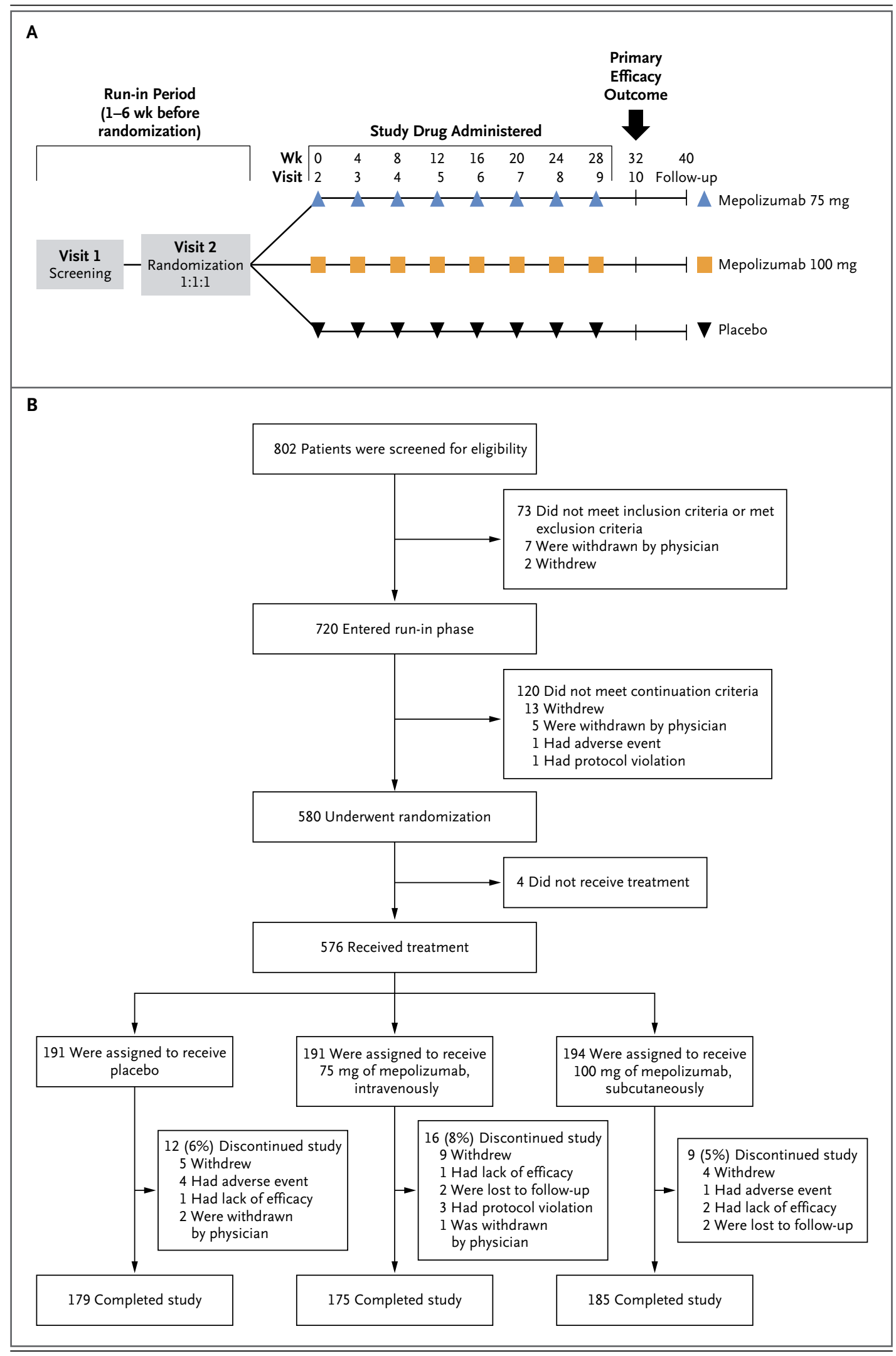

N ENGLJ MED 371;13 NEJM.ORG SEPTEMBER 25, 2014

The New England Journal of Medicine

Downloaded from nejm.org by R LOUIS SPRL on January 16, 2015. For personal use only. No other uses without permission. Copyright () 2014 Massachusetts Medical Society. All rights reserved. 
fied intention-to-treat population; of these patients, 539 (94\%) completed treatment (Fig. 1B). The study groups were well matched with respect to baseline characteristics, with no significant differences among the groups (Table 1).

\section{PRIMARY OUTCOME}

A total of 446 objectively verified exacerbations that led to the use of systemic glucocorticoids, hospitalization, or an emergency department visit were reported. The rate of exacerbations was lower than the rate at baseline in all groups, but the percentage reduction was greater in the activetreatment groups than in the placebo group. The estimated rates of clinically significant exacerbations per patient per year were 0.93 in the intravenous-mepolizumab group, 0.81 in the subcutaneous-mepolizumab group, and 1.75 in the placebo group. As compared with placebo, the relative reduction in exacerbation rate (primary outcome) was $47 \%$ ( $95 \%$ confidence interval [CI], 29 to 61) in the intravenous-mepolizumab group and $53 \%$ (95\% CI, 37 to 65 ) in the subcutaneousmepolizumab group ( $\mathrm{P}<0.001$ for both comparisons) (Table 2).

The proportion of patients with an exacerbation that resulted in an emergency department visit or hospitalization was $9 \%$ in the intravenous-mepolizumab group, $6 \%$ in the subcutaneous-mepolizumab group, and $13 \%$ in the placebo group. The use of mepolizumab resulted in a relative reduction in the rate of exacerbations requiring hospitalization or an emergency department visit of $32 \%$ in the intravenous-mepolizumab group $(\mathrm{P}=0.30)$ and $61 \%$ in the subcutaneous-mepolizumab group $(\mathrm{P}=0.02)$. Similar reductions were seen in mepolizumab-treated groups with respect to exacerbations resulting in hospitalization (39\% and 69\%, respectively) (Table 2). The cumulative number of exacerbations over time is shown in Figure 2A. Data regarding the time to the first exacerbation and the mean duration of exposure to systemic glucocorticoids are provided in Figures S1 and S2 in the Supplementary Appendix.

\section{SECONDARY OUTCOMES AND OTHER MEASURES Lung Function}

At week 32, the mean increase from baseline in $\mathrm{FEV}_{1}$ before bronchodilation was $100 \mathrm{ml}$ greater in the intravenous-mepolizumab group than in the placebo group $(\mathrm{P}=0.02)$ and $98 \mathrm{ml}$ greater in the subcutaneous-mepolizumab group than in the pla- cebo group ( $\mathrm{P}=0.03)$ (Table 2 and Fig. 2B). The mean increase from baseline in $\mathrm{FEV}_{1}$ after bronchodilation was $146 \mathrm{ml}$ greater in the intravenous-mepolizumab group than in the placebo group $(\mathrm{P}=0.003)$ and $138 \mathrm{ml}$ greater in the subcutaneous-mepolizumab group than in the placebo group ( $\mathrm{P}=0.004)$ (Table 2). At week 32, the daily morning peak expiratory flow rate increased by 22.9 liters per minute in the intravenous-mepolizumab group, by 29.5 liters per minute in the subcutaneous-mepolizumab group, and by $1.8 \mathrm{li}$ ters per minute in the placebo group.

\section{Quality of Life}

At week 32, patients in the two mepolizumab groups had a significant improvement (i.e., a numerical decrease) in SGRQ total scores, as compared with patients in the placebo group. The reductions from baseline in total scores were 6.4 points greater in the intravenous-mepolizumab group and 7.0 points greater in the subcutaneousmepolizumab group than in the placebo group ( $\mathrm{P}<0.001$ for both comparisons) (Table 2, and Fig. S3 in the Supplementary Appendix).

\section{Asthma Control}

At baseline, patients in the three study groups had similar mean ACQ-5 scores (2.12 in the intravenous-mepolizumab group, 2.26 in the subcutaneous-mepolizumab group, and 2.28 in the placebo group), indicating uncontrolled asthma. As early as week 4 and continuing through week 32, patients in the two mepolizumab groups had greater improvement (i.e., a numerical decrease) from baseline in ACQ-5 scores, as compared with placebo. At week 32, the mean reductions in total scores were 0.42 points greater in the intravenous-mepolizumab group and 0.44 points greater in the subcutaneous-mepolizumab group than in the placebo group ( $\mathrm{P}<0.001$ for both comparisons) (Table 2, and Fig. S4 in the Supplementary Appendix).

Responses as Rated by Patients and Clinicians At week 32, patients in the two mepolizumab groups gave higher marks to therapy than did patients in the placebo group, with odds ratios of 1.74 in the intravenous-mepolizumab group $(\mathrm{P}=0.003)$ and 2.98 in the subcutaneous-mepolizumab group $(\mathrm{P}<0.001)$ (with values of more than 1 indicating a greater probability that a patient recorded a higher response for mepolizumab than for placebo). 


\begin{tabular}{|c|c|c|c|}
\hline \multirow[t]{2}{*}{ Characteristic } & \multirow[t]{2}{*}{$\begin{array}{l}\text { Placebo } \\
(\mathrm{N}=191)\end{array}$} & \multicolumn{2}{|c|}{ Mepolizumab } \\
\hline & & $\begin{array}{c}\text { Intravenous } \\
(\mathrm{N}=191)\end{array}$ & $\begin{array}{l}\text { Subcutaneous } \\
\qquad(N=194)\end{array}$ \\
\hline Mean age (range) $-\mathrm{yr}$ & $49(12-76)$ & $50(13-82)$ & $51(12-81)$ \\
\hline Female sex — no. (\%) & $107(56)$ & $106(55)$ & $116(60)$ \\
\hline Body-mass index $†$ & $28.0 \pm 5.6$ & $27.7 \pm 5.7$ & $27.6 \pm 6.2$ \\
\hline Former smoker — no. (\%) & $57(30)$ & $52(27)$ & $50(26)$ \\
\hline Duration of asthma $-y r$ & $19.5 \pm 14.6$ & $19.8 \pm 14.0$ & $20.5 \pm 12.9$ \\
\hline \multicolumn{4}{|l|}{ Use of oral glucocorticoids } \\
\hline Maintenance use - no. (\%) & $44(23)$ & $48(25)$ & $52(27)$ \\
\hline Mean daily dose (range) - mgt & $15.1(5-80)$ & $12.0(1-40)$ & $12.6(2-50)$ \\
\hline Allergic rhinitis - no. (\%) & $95(50)$ & $91(48)$ & $95(49)$ \\
\hline \multicolumn{4}{|l|}{$\mathrm{FEV}_{1}$} \\
\hline Before bronchodilation — liters $\rrbracket$ & $1.86 \pm 0.63$ & $1.86 \pm 0.70$ & $1.73 \pm 0.66$ \\
\hline Percent of predicted value before bronchodilation & $62.4 \pm 18.1$ & $61.4 \pm 18.3$ & $59.3 \pm 17.5$ \\
\hline Reversibility — \% & $27.4 \pm 20.8$ & $25.4 \pm 19.6$ & $27.9 \pm 24.0$ \\
\hline $\mathrm{FEV}_{1}: \mathrm{FVC}$ ratio - \% $\|$ & $64 \pm 13$ & $64 \pm 13$ & $63 \pm 13$ \\
\hline Morning peak expiratory flow - liters/min & $277 \pm 106$ & $269 \pm 112$ & $255 \pm 108$ \\
\hline Score on Asthma Control Questionnaire*** & $2.28 \pm 1.19$ & $2.12 \pm 1.13$ & $2.26 \pm 1.27$ \\
\hline Score on St. George's Respiratory Questionnaire'†' & $46.9 \pm 19.8$ & $44.4 \pm 19.4$ & $47.9 \pm 19.4$ \\
\hline Geometric mean IgE on $\log _{\mathrm{e}}$ scale $-U / \mathrm{ml}$ & $150 \pm 1.5$ & $180 \pm 1.5$ & $150 \pm 1.5$ \\
\hline Geometric mean blood eosinophil count on $\log _{\mathrm{e}}$ scale - cells $/ \mu \mid t+$ & $320 \pm 938$ & $280 \pm 987$ & $290 \pm 1050$ \\
\hline \multicolumn{4}{|l|}{ Asthma exacerbations } \\
\hline Severe episodes in previous year - no./patient & $3.6 \pm 2.8$ & $3.5 \pm 2.2$ & $3.8 \pm 2.7$ \\
\hline Necessitating hospitalization in previous year — no. (\%) & $35(18)$ & $41(21)$ & $33(17)$ \\
\hline History of asthma-related intubation — no. (\%) & $3(2)$ & $10(5)$ & $8(4)$ \\
\hline
\end{tabular}

* Plus-minus values are means (or geometric means) \pm SD. There were no significant between-group differences at baseline. More detailed data are provided in Table S3 in the Supplementary Appendix. FEV ${ }_{1}$ denotes forced expiratory volume in 1 second, and FVC forced vital capacity.

$\dagger$ The body-mass index is the weight in kilograms divided by the square of the height in meters.

The listed value is the prednisone equivalent.

$\int$ Reversibility was measured at baseline.

I The percent of the predicted value before bronchodilation was assessed at the screening visit.

$\|$ The $\mathrm{FEV}_{1}$ : $\mathrm{FVC}$ ratio was calculated by dividing the $\mathrm{FEV}$ by the $\mathrm{FVC}$ and then multiplying by 100 to express the value as a percentage.

** Scores on the Asthma Control Questionnaire range from 0 to 6, with higher scores indicating worse control; a change of 0.5 points is the minimal clinically important difference.

†† Scores on St. George's Respiratory Questionnaire range from 0 to 100, with higher scores indicating worse function; a change of 4 points is considered to be clinically relevant.

$+t$ Values below the lower limit of quantification (LLQ) were replaced by a value that was $50 \%$ of the LLQ.

The corresponding odds ratios in the physicianrated overall evaluation of response to therapy were 2.10 for intravenous mepolizumab and 3.29 for subcutaneous mepolizumab $(\mathrm{P}<0.001$ for both comparisons) (Table S2 and Fig. S5 in the Supplementary Appendix).

\section{Blood Eosinophil Count}

Blood eosinophil counts were similar in the three groups at baseline, with a geometric mean of 295 cells per microliter (Table 1). Mepolizumab decreased the eosinophil counts by week 4; the counts reached a nadir around week 12 (with re- 


\begin{tabular}{|c|c|c|c|c|c|c|c|}
\hline Outcome & $\begin{array}{l}\text { Placebo } \\
(\mathrm{N}=191)\end{array}$ & $\begin{array}{l}\text { Intravenous } \\
\text { Mepolizumab } \\
(\mathrm{N}=191)\end{array}$ & $\begin{array}{l}\text { Difference } \\
\text { from Placebo } \\
(95 \% \mathrm{Cl})\end{array}$ & $\begin{array}{c}\mathbf{P} \\
\text { Value }\end{array}$ & $\begin{array}{l}\text { Subcutaneous } \\
\text { Mepolizumab } \\
(N=194)\end{array}$ & $\begin{array}{l}\text { Difference } \\
\text { from Placebo } \\
(95 \% \mathrm{Cl})\end{array}$ & $\begin{array}{c}P \\
\text { Value }\end{array}$ \\
\hline $\begin{array}{c}\text { Mean rate of clinically significant } \\
\text { exacerbations }\end{array}$ & 1.75 & 0.93 & $47(29$ to 61$) \dagger$ & $<0.001$ & 0.81 & $53(37$ to 65$) \dagger$ & $<0.001$ \\
\hline $\begin{array}{l}\text { Mean rate of exacerbations re- } \\
\text { quiring hospitalization or } \\
\text { emergency department } \\
\text { visit }\end{array}$ & 0.20 & 0.14 & $32(-41$ to 67$) \dagger$ & 0.30 & 0.08 & 61 (17 to 82$) \dagger$ & 0.02 \\
\hline $\begin{array}{c}\text { Mean rate of exacerbations re- } \\
\text { quiring hospitalization }\end{array}$ & 0.10 & 0.06 & $39(-66$ to 77$) \dagger$ & 0.33 & 0.03 & $69(9$ to 89$) \uparrow$ & 0.03 \\
\hline \multicolumn{8}{|l|}{ Change from baseline in $\mathrm{FEV}_{1}-\mathrm{ml}$} \\
\hline Before bronchodilation & $86 \pm 31$ & $186 \pm 32$ & 100 (13 to 187$)$ & 0.02 & $183 \pm 31$ & 98 (11 to 184$)$ & 0.03 \\
\hline After bronchodilation & $30 \pm 34$ & $176 \pm 34$ & 146 (50 to 242$)$ & 0.003 & $167 \pm 33$ & 138 (43 to 232$)$ & 0.004 \\
\hline $\begin{array}{c}\text { Change from baseline in score } \\
\text { on Asthma Control } \\
\text { Questionnaire }\end{array}$ & $-0.50 \pm 0.07$ & $-0.92 \pm 0.07$ & $-0.42(-0.61$ to -0.23$)$ & $<0.001$ & $-0.94 \pm 0.07$ & $-0.44(-0.63$ to -0.25$)$ & $<0.001$ \\
\hline $\begin{array}{l}\text { Change from baseline in score on } \\
\text { St. George's Respiratory } \\
\text { Questionnaire }\end{array}$ & $-9.0 \pm 1.2$ & $-15.4 \pm 1.2$ & $-6.4(-9.7$ to -3.2$)$ & $<0.001$ & $-16.0 \pm 1.1$ & $-7.0(-10.2$ to -3.8$)$ & $<0.001$ \\
\hline
\end{tabular}

$*$ Plus-minus values are means \pm SE.

$\uparrow$ The between-group difference in this category is the percent reduction as compared with the placebo group.

ductions of $83 \%$ in the intravenous group and $86 \%$ in the subcutaneous group), and the decreases were maintained during the study (Fig. S6 in the Supplementary Appendix).

\section{SUBGROUP ANALYSIS}

Modeling of baseline characteristics to determine the effect of individual covariates is shown in Figure S7 in the Supplementary Appendix. To further evaluate the model, we performed a prespecified analysis of clinical end points in a subgroup of patients with a blood eosinophil count of 500 cells per microliter or more (Fig. S8 in the Supplementary Appendix). This subgroup analysis showed an enhanced response to mepolizumab.

\section{SAFETY}

The overall incidence of adverse events during treatment was similar in the three groups $(84 \%$ in the intravenous-mepolizumab group, $78 \%$ in the subcutaneous-mepolizumab group, and $83 \%$ in the placebo group). The most frequently reported adverse events were nasopharyngitis and headache. The incidence of adverse events that were considered by the study investigators to be related to a study drug was $17 \%$ in the intravenous-mepolizumab group, $20 \%$ in the subcutaneous-mepoliz- umab group, and $16 \%$ in the placebo group. The incidence of injection-site reactions was more frequent in the subcutaneous-mepolizumab group $(9 \%)$ than in the intravenous-mepolizumab group or the placebo group (3\% in both groups) (Table 3). The incidence of serious adverse events (including asthma-related events) was $7 \%$ in the intravenous-mepolizumab group, $8 \%$ in the subcutaneous-mepolizumab group, and $14 \%$ in the placebo group. One death due to a road-traffic accident occurred in a 51-year-old man in the placebo group (Table 3). During testing at least one visit after randomization, positive anti-mepolizumab antibodies were found in 19 patients, including $4 \%$ of the patients in the intravenous-mepolizumab group, $5 \%$ of those in the subcutaneousmepolizumab group, and $2 \%$ of those in the placebo group. None of these patients had neutralizing antibodies.

\section{DISCUSSION}

In patients treated with high-dose inhaled oral glucocorticoids with or without maintenance oral glucocorticoids for asthma control, treatment with mepolizumab reduced exacerbations by approximately one half, improved quality of life, and re- 
sulted in better asthma control. Both intravenous and subcutaneous doses were effective and had acceptable side-effect profiles. In a previous study, ${ }^{11}$ mepolizumab did not produce a significant benefit, but patients were not selected on the basis of frequent exacerbations, treatment with high doses of inhaled glucocorticoids, or a specific blood eosinophil count. Using a specific hematologic and phenotyping approach for patient selection, we confirmed that neutralizing interleukin-5 with mepolizumab can be effective in a subpopulation of patients with severe eosinophilic asthma. ${ }^{1,2,4,18}$

In this study, we used a closed-testing procedure to adjust for multiple comparisons with respect to the primary and secondary outcomes. Differences in the rates of exacerbations (the primary outcome) in the two mepolizumab groups, as compared with the placebo group, were significant after adjustment. Among patients receiving subcutaneous mepolizumab, the reduction in the rate of exacerbations resulting in an emergency department visit or hospitalization (a secondary outcome) was significant after adjustment for multiple testing $(\mathrm{P}=0.03)$. Although the hierarchical gatekeeping approach across outcomes dictated that formal analysis was to be stopped before analysis of the remaining secondary outcomes, the value of such adjustments has been questioned. Instead, it has been proposed that expert judgment should be used for the interpretation of secondary outcomes. ${ }^{19}$

Since $94 \%$ of the patients in our study elected to participate in an open-label extension study, there is a paucity of data characterizing clinical status at the time of the cessation of treatment. However, in a 12-month observational study evaluating the response to the cessation of mepolizumab, Haldar and colleagues ${ }^{20}$ found that eosinophil counts in blood and sputum increased significantly, returning to pretreatment values within 3 months after mepolizumab was stopped. This change was associated with a loss of asthma control, and 3 to 6 months after cessation of treatment, patients were found to have pretreatment exacerbation levels.

The main difference between our study and the companion study reported in the Journal by Bel and colleagues $^{14}$ is the rate of use of daily oral glucocorticoids ( $25 \%$ and $100 \%$, respectively). Physicians strive to reduce both the frequency and dose of glucocorticoids while maintaining asthma control. These two studies provide insight on the
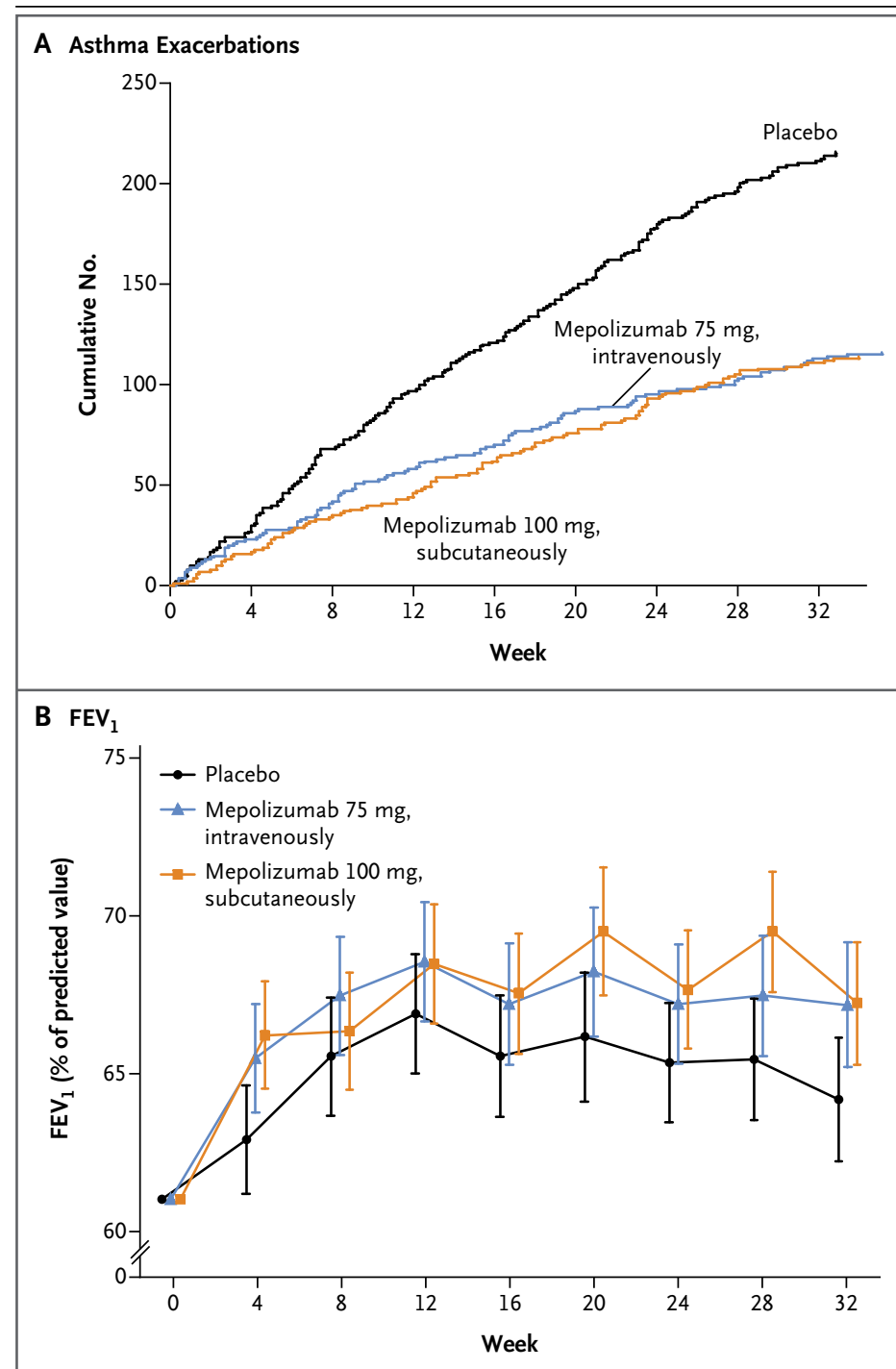

Figure 2. Asthma Exacerbations and $\mathrm{FEV}_{\mathbf{1}}$ at $\mathbf{3 2}$ Weeks.

Panel A shows the numbers of asthma exacerbations in patients receiving either intravenous or subcutaneous mepolizumab or placebo. The rate of exacerbations was reduced by $47 \%$ (95\% confidence interval [CI], 29 to 61 ) among patients receiving intravenous mepolizumab and by $53 \%(95 \% \mathrm{Cl}$, 37 to 65 ) among those receiving subcutaneous mepolizumab, as compared with those receiving placebo $(\mathrm{P}<0.001$ for both comparisons). Panel $B$ shows the mean forced expiratory volume in 1 second $\left(F^{2} V_{1}\right)$ as a percentage of the predicted value. At week 32, there was greater improvement from baseline in the two mepolizumab groups than in the placebo group - a 100-ml greater increase in the intravenous-mepolizumab group than in the placebo group $(P=0.02)$ and a $98-\mathrm{ml}$ greater increase in the subcutaneous-mepolizumab group than in the placebo group $(P=0.03)$. The I bars indicate $95 \%$ confidence intervals.

concept that asthma control and improvement can be achieved in patients meeting our phenotypic definition. Overall, our study further confirms the benefit of mepolizumab in the reduction of exac- 


\begin{tabular}{|c|c|c|c|}
\hline \multirow[t]{3}{*}{ Variable } & \multirow[t]{2}{*}{$\begin{array}{l}\text { Placebo } \\
(\mathrm{N}=191)\end{array}$} & \multicolumn{2}{|c|}{ Mepolizumab } \\
\hline & & $\begin{array}{c}\text { Intravenous } \\
(\mathrm{N}=191)\end{array}$ & $\begin{array}{l}\text { Subcutaneous } \\
(N=194)\end{array}$ \\
\hline & \multicolumn{3}{|c|}{ number of patients (percent) } \\
\hline All adverse events & $158(83)$ & $161(84)$ & $152(78)$ \\
\hline Nonasthma event & $157(82)$ & $161(84)$ & $152(78)$ \\
\hline Worsening of asthma & $29(15)$ & $18(9)$ & $13(7)$ \\
\hline Drug-related event, per investigator assessment $\dagger$ & $30(16)$ & $33(17)$ & $39(20)$ \\
\hline Leading to study withdrawal & $4(2)$ & 0 & $1(1)$ \\
\hline \multicolumn{4}{|l|}{ Serious adverse events } \\
\hline During treatment & $27(14)$ & $14(7)$ & $16(8)$ \\
\hline Drug-related event, per investigator assessment $\dagger$ & $1(1)$ & 0 & $1(1)$ \\
\hline Fatal & $1(1)$ & 0 & 0 \\
\hline \multicolumn{4}{|l|}{ Most common adverse events $\downarrow$} \\
\hline Nasopharyngitis & $46(24)$ & $45(24)$ & $33(17)$ \\
\hline Headache & $33(17)$ & $46(24)$ & $39(20)$ \\
\hline Upper respiratory tract infection & $27(14)$ & $22(12)$ & $24(12)$ \\
\hline Sinusitis & $18(9)$ & $11(6)$ & $18(9)$ \\
\hline Bronchitis & $18(9)$ & $14(7)$ & $9(5)$ \\
\hline Oropharyngeal pain & $15(8)$ & $12(6)$ & $7(4)$ \\
\hline Injection-site reaction & $6(3)$ & $5(3)$ & $17(9)$ \\
\hline
\end{tabular}

* A more detailed listing of adverse events is provided in Table S4 in the Supplementary Appendix. $\uparrow$ The status was assigned by investigators while they were unaware of the study-group assignments. $\lceil$ The most common adverse events were those that were reported in at least $5 \%$ of the patients in any study group.

erbations and expands on the benefits with respect to quality of life and other markers of asthma control in patients identified according to clinical characteristics and blood eosinophil counts.

Supported by GlaxoSmithKline.
Disclosure forms provided by the authors are available with the full text of this article at NEJM.org.

We thank the patients and the research teams for their participation in this study; and Robert Price, Janet Perkins, and Cilla Mistry for their support in data collection and analysis.

\section{REFERENCES}

1. Custovic A, Johnston SL, Pavord I, et al. EAACI position statement on asthma exacerbations and severe asthma. Allergy 2013;68:1520-31.

2. Omachi TA, Iribarren C, Sarkar U, et al. Risk factors for death in adults with severe asthma. Ann Allergy Asthma Immunol 2008;101:130-6.

3. Cisternas MG, Blanc PD, Yen IH, et al. A comprehensive study of the direct and indirect costs of adult asthma. J Allergy Clin Immunol 2003;111:1212-8.

4. Chung KF, Wenzel SE, Brozek JL, et al. International ERS/ATS guidelines on definition, evaluation and treatment of severe asthma. Eur Respir J 2014;43:34373.
5. Wenzel SE. Asthma: defining of the persistent adult phenotypes. Lancet 2006; 368:804-13.

6. Wenzel SE. Asthma phenotypes: the evolution from clinical to molecular approaches. Nat Med 2012;18:716-25.

7. Flood-Page P, Menzies-Gow A, Phipps $\mathrm{S}$, et al. Anti-IL-5 treatment reduces deposition of ECM proteins in the bronchial subepithelial basement membrane of mild atopic asthmatics. J Clin Invest 2003;112: 1029-36.

8. Menzies-Gow A, Flood-Page P, Sehmi $\mathrm{R}$, et al. Anti-IL-5 (mepolizumab) therapy induces bone marrow eosinophil maturational arrest and decreases eosinophil progenitors in the bronchial mucosa of atopic asthmatics. J Allergy Clin Immunol 2003;111:714-9.

9. Flood-Page P, Swenson C, Faiferman I, et al. A study to evaluate safety and efficacy of mepolizumab in patients with moderate persistent asthma. Am J Respir Crit Care Med 2007;176:1062-71.

10. Haldar P, Brightling CE, Hargadon B, et al. Mepolizumab and exacerbations of refractory eosinophilic asthma. $\mathrm{N}$ Engl J Med 2009;360:973-84.

11. Nair P, Pizzichini MM, Kjarsgaard M, et al. Mepolizumab for prednisonedependent asthma with sputum eosinophilia. N Engl J Med 2009;360:985-93.

12. Leckie MJ, ten Brinke A, Khan J, et al. Effects of an interleukin-5 blocking 
monoclonal antibody on eosinophils, airway hyper-responsiveness, and the late asthmatic response. Lancet 2000;356: 2144-8.

13. Pavord ID, Korn S, Howarth P, et al. Mepolizumab for severe eosinophilic asthma (DREAM): a multicentre, doubleblind, placebo-controlled trial. Lancet 2012;380:651-9.

14. Bel EH, Wenzel SE, Thompson PJ, et al. Oral glucocorticoid-sparing effect of mepolizumab in eosinophilic asthma. $\mathrm{N}$ Engl Med 2014;371:1189-97.

15. Reddel HK, Taylor DR, Bateman ED, et al. An official American Thoracic Soci-
ety/European Respiratory Society statement: asthma control and exacerbations: standardizing endpoints for clinical asthma trials and clinical practice. Am J Respir Crit Care Med 2009;180:59-99.

16. Keene ON, Calverley PM, Jones PW, Vestbo J, Anderson JA. Statistical analysis of exacerbation rates in COPD: TRISTAN and ISOLDE revisited. Eur Respir J 2008; 32:17-24.

17. Keene ON, Jones MR, Lane PW, Anderson J. Analysis of exacerbation rates in asthma and chronic obstructive pulmonary disease: example from the TRISTAN study. Pharm Stat 2007;6:89-97.
18. Chanez P, Wenzel SE, Anderson GP, et al. Severe asthma in adults: what are the important questions? J Allergy Clin Immunol 2007;119:1337-48.

19. Stone A, Chuang-Stein C. Strong control over multiple endpoints: are we adding value to the assessment of medicines? Pharm Stat 2013;12:189-91.

20. Haldar P, Brightling CE, Singapuri A, et al. Outcomes after cessation of mepolizumab therapy in severe eosinophilic asthma: a 12-month follow-up analysis. J Allergy Clin Immunol 2014;133:921-3. Copyright (c) 2014 Massachusetts Medical Society.

The New England Journal of Medicine 